\title{
The assessment of general movements
} in term and late-preterm infants diagnosed with neonatal encephalopathy, as a predictive tool of cerebral palsy by 2 years of age-a scoping review

Judy Seesahai ${ }^{1}$, Maureen Luther ${ }^{1}$, Paige Terrien Church ${ }^{1,2}$, Patricia Maddalena', Elizabeth Asztalos ${ }^{1,2}$,

Thomas Rotter ${ }^{3}$ and Rudaina Banihani $i^{1,2,4^{*}}$ (D)

\begin{abstract}
Background: The General Movements Assessment is a non-invasive and cost-effective tool with demonstrated reliability for identifying infants at risk for cerebral palsy. Early detection of cerebral palsy allows for the implementation of early intervention and is associated with better functional outcomes. No review to date has summarized the utility of the General Movements Assessment to predict cerebral palsy in term and late-preterm infants diagnosed with neonatal encephalopathy.
\end{abstract}

Methods: We conducted a scoping review involving infants born greater than or equal to 34 weeks gestational age to identify all available evidence and delineate research gaps. We extracted data on sensitivity, specificity, and positive and negative predictive values and described the strengths and limitations of the results. We searched five databases (MEDLINE, Embase, PsychINFO, Scopus, and CINAHL) and the General Movements Trust website. Two reviewers conducted all screening and data extraction independently. The articles were categorized according to key findings, and a critical appraisal was performed.

Results: Only three studies, a cohort and two case series, met all of the inclusion criteria. The total number of participants was 118. None of the final eligible studies included late-preterm neonates. All three studies reported on sensitivity, specificity, and positive predictive and negative predictive values. An abnormal General Movement Assessment at 3-5 months has a high specificity (84.6-98\%) for cerebral palsy with a similarly high negative predictive value (84.6-98\%) when it was normal. Absent fidgety movements, in particular, are highly specific (96\%) for moderate to severe cerebral palsy and carry a high negative predictive value (98\%) when normal. In the time period between term and 4-5 months post-term, any cramped synchronized movements had results of 100\% sensitivity and variable results for specificity, positive predictive value, and negative predictive value.

Conclusions: A normal General Movements Assessment at 3 months in a term high-risk infant is likely associated with a low risk for moderate/severe cerebral palsy. The finding of cramped synchronized General Movements is a

\footnotetext{
*Correspondence: rudaina.banihani@sunnybrook.ca

${ }^{4}$ Newborn \& Developmental Paediatrics, Sunnybrook Health Science

Centre, 2075, Bayview Ave., Toronto, ON M4N 3M5, Canada

Full list of author information is available at the end of the article
}

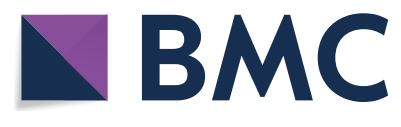

(c) The Author(s) 2021. Open Access This article is licensed under a Creative Commons Attribution 4.0 International License, which permits use, sharing, adaptation, distribution and reproduction in any medium or format, as long as you give appropriate credit to the original author(s) and the source, provide a link to the Creative Commons licence, and indicate if changes were made. The images or other third party material in this article are included in the article's Creative Commons licence, unless indicated otherwise in a credit line to the material. If material is not included in the article's Creative Commons licence and your intended use is not permitted by statutory regulation or exceeds the permitted use, you will need to obtain permission directly from the copyright holder. To view a copy of this licence, visit http://creativecommons.org/licenses/by/4.0/. The Creative Commons Public Domain Dedication waiver (http://creativeco mmons.org/publicdomain/zero/1.0/) applies to the data made available in this article, unless otherwise stated in a credit line to the data. 
strong predictor for the diagnosis of cerebral palsy by 2 years of age in the term population with neonatal encephalopathy. The deficit of high-quality research limits the applicability, and so the General Movements Assessment should not be used in isolation when assessing this population.

Systematic review registration: Title registration with Joanna Briggs Institute. URL: http://joannabriggswebdev.org/ research/registered_titles.aspx.

Keywords: Neonatal encephalopathy, General Movement Assessment, Prechtl, Hypoxia-ischemia encephalopathy, Cerebral palsy, Infants, Neonates, Term babies, Preterm babies, Motor development

\section{Background}

Cerebral palsy (CP) is defined by the Executive Committee for the Definition of Cerebral Palsy in 2006 as "a group of disorders of the development of movement and posture, causing activity limitation, that are attributed to non-progressive disturbances that occurred in the developing fetal or infant brain" [1]. Cerebral palsy is known to be a potential outcome of perinatal asphyxia, with at-risk neonates presenting with encephalopathy, specifically hypoxic-ischemic encephalopathy (HIE) [2]. The American Academy of Pediatrics and the American College of Obstetrics and Gynecology have outlined specific criteria for the diagnosis of HIE [2], and there is strong evidence for therapeutic hypothermia $[3,4]$ for those term or latepreterm presenting with HIE. Therapeutic hypothermia is shown to improve survival and the 18-month neurodevelopmental outcomes [5], including CP, but it does not completely eradicate the possibility of long-term neurodevelopmental disability [6].

While therapeutic hypothermia can mitigate any injury associated with HIE, early identification and intervention of $\mathrm{CP}$ are imperative as there is now strong evidence demonstrating effective tools for the identification and improved functional outcomes associated with a targeted intervention. Prognostication of long-term neurodevelopmental outcomes has utilized a combination of clinical and radiological tools $[7,8]$. One tool that has emerged as a strong predictor of neurological integrity is the General Movements Assessment (GMA), developed by Dr. Heinz Prechtl first in $1979[9,10]$. This assessment describes the repertoire of complex, highly variable, whole-body movements which emerge in the fetus and continue until the first 4 to 5 months of life $[11,12]$. Specific patterns exist at set developmental stages (preterm, writhing, and fidgety), and patterns of stereotypy have also been identified that are associated with CP $[13,14]$. Various scoring algorithms have been developed with Prechtl and HaddersAlgra [14] being the most reported.

All reviews to date have focused on an amalgamated population of at-risk infants, combining those born preterm as well as those term and late-preterm infants, most recently in two systematic reviews from 2017 by Novak et al. [15] and 2018 by Kwong et al. [16]. There have been another eight reviews completed between 2001 and 2013; of these, seven were systematic reviews [12, 17-22] and one literature review [23]. There are two reviews around the topic of the predictive value of the GMA pending [24, $25]$, both of which are systematic reviews.

The key characteristics and main findings of the above reviews on GMA are detailed in the protocol for this systematic review [26] and are included in Additional file 1: Table 1. The systematic reviews from 2017 [15] and 2018 [16] looked at a variety of assessments in early infancy utilized to predict CP, including the GMA, the Hammersmith Infant Neurological Examination (HINE) [27], the Movement Assessment of Infants (MAI) [28], and magnetic resonance imaging (MRI). The 2017 review demonstrated that the GMA had the highest sensitivity (98\%) for CP [15]. The 2018 review demonstrated a high sensitivity of 93\% (95\% confidence interval (CI) 86-96) but a low specificity for CP $59 \%$ (95\% CI 45-71) with the GMA (using Prechtl scoring algorithm) at 6 weeks (writhing phase). Later assessments (10-20 weeks), from the fidgety period (evaluating for the presence and quality of fidgety movements (FMs)), however, demonstrated a better sensitivity of 97\% (CI 95\% 93-99) and a specificity of $89 \%$ (95\% CI 83-93) [16]. The conclusion was those later assessments in infancy with the quality of FMs as assessed by GMA had the strongest predictive value for CP.

To date, there are no current reviews that exist describing the predictive value of GMA in a population of nearterm or term neonates presenting with encephalopathy. Based on this gap in the literature, we aim to conduct a scoping review on this specific population, as the first priority was to determine the type and extent of available evidence. The key concepts as it relates to the GMA and its use in this and term late-preterm population need to be clarified. Completion of this scoping review will determine the feasibility of a systematic review and meta-analysis.

\section{Objectives}

The primary research question for this review is: What is the published data on the predictive value of the GMA 
for the diagnosis of CP by 2 to 3 years of age in infants born at term or late-preterm presenting with NE?

The secondary research question is: What is the gap in the literature when the GMA is used to predict CP by 2 years of age in infants born at term or late-preterm presenting with NE?

\section{Methods}

\section{Study design}

A scoping method was chosen for this type of review to fulfill our objective which requires searching and assessing a wide range of research methodologies involving the use of the GMA in CP prediction. A scoping review captured all types of relevant research on the topic in a systematic, transparent, rigorous, and reproducible manner. This scoping review was conducted in accordance with the JBI methodology for scoping reviews [29]. The objectives, inclusion criteria, and methods for this scoping review were detailed in advance and documented in a proposal (included as Additional file 2). The title of our review was registered with JBI. The protocol was published prior to this review [26] (Additional file 1).

Inherent in the nature of the scoping review is the inclusiveness of a wide range of literature, and so, we anticipated the differences in the data quality. Critical appraisal and data synthesis therefore were challenging in terms of conclusive evidence as opposed to that from a systematic review. The scoping review methodology was however especially advantageous to our question as these types of reviews target areas that have not been comprehensively assessed before.

\section{Eligibility criteria}

The participant, concept, context (PCC) framework for scoping reviews was used to define the review focus and is summarized in Table 1 below (see Additional file 1).

\section{Participants}

Our population of interest was those born at $\geq 34$ 0/7 weeks GA, and we defined this population based on several factors. First, there are already systematic reviews that have consolidated the evidence in the preterm populations for the use of the GMA in the prediction of neurodevelopmental outcomes, but this does not exist for the older gestational age groups $[15,16]$. Secondly, the benefit of therapeutic hypothermia for NE caused by HIE is established in infants $\geq 35$ weeks GA, and ongoing research considers the extension of therapeutic hypothermia treatment to a GA younger than 35 weeks [4, 5, 30].

We therefore chose to focus on the population of $\geq 34$ 0/7 weeks GA, firstly to comprehensively consolidate this body of literature. Secondly, we will lay the foundation for future studies looking at the utility of the GMA in the prediction of $\mathrm{CP}$ before and after the introduction of therapeutic hypothermia.

\section{Concept}

The GMA is a non-invasive tool that has been shown to have predictive validity for $\mathrm{CP}^{6}$. Traditionally, $\mathrm{CP}$ has been diagnosed by 12 to 24 months [1, 31, 32]. We considered a diagnosis by 2 years as by this time, the majority of diagnoses should be made [14]. Given the practicality of obtaining an assessment for $\mathrm{CP}$ at precisely less than or equal to 24 months, we have decided to use the time frame of 2-3 years. We appreciate that milder forms of $\mathrm{CP}$ may be diagnosed at ages older than 2 years of age. The stability of the diagnosis of $\mathrm{CP}$ is also traditionally better at the older ages. Our aim however was to consolidate the literature for the age at which CP is most likely diagnosed [31-36]. Additionally, in term survivors of hypoxic-ischemic brain injury, spastic quadriparesis is the most common type of CP, although athethoid

Table 1 Inclusion and exclusion criteria for the prediction of CP by the GMA in late-preterm and term infants with NE

\begin{tabular}{|c|c|c|}
\hline & Inclusion criteria & Exclusion criteria \\
\hline Participants & $\begin{array}{l}\text { Studies with infants who were: } \\
\text { - } \geq 340 / 7 \text { wks GA } \\
\text { - Diagnosis of NE } \\
\text { - Had a GMA done between birth up to } 6 \text { months of life } \\
\text { - had an assessment for CP by at least } 2 \text { years of age }\end{array}$ & $\begin{array}{l}\text { Studies in which a diagnosis of CP was made after } 2-3 \text { years of age } \\
\text { Studies with infants born with life-threatening congenital abnor- } \\
\text { malities, congenital viral infections, an abnormal karyotype, and } \\
\text { metabolic disorders } \\
\text { Animal studies }\end{array}$ \\
\hline Concept & GMA as a predictor of CP by 2 years of age is the main concept & \\
\hline Context & $\begin{array}{l}\text { Studies that reported on: } \\
\text { - Infants with NE managed and diagnosed by the standard of } \\
\text { care (neurological history and examination) } \\
\text { - Studies from all countries that have outcomes reported in the } \\
\text { acute neonatal and in the follow-up period by } 2 \text { years of age } \\
\text { - Studies in the English language only }\end{array}$ & $\begin{array}{l}\text { Text and opinion papers were not considered for inclusion as this is a } \\
\text { highly specific and medical topic }\end{array}$ \\
\hline
\end{tabular}

CP cerebral palsy, GA gestational age, GMA general movements assessment, NE neonatal encephalopathy, wks weeks 
or spastic hemiparesis also occurs which would likely be evident by $2-3$ years [37-40].

For the GMA to be used as a predictive tool for CP, we considered studies which measured sensitivity, specificity, positive predictive value, and negative predictive value as detailed in our protocol. These measurements provide the best guidance for this clinical application [41]. Detailed definitions of concepts can be found in Additional file 1: Table 3.

In terms of the type of GMA, we collected studies using any type of GMA although we are aware that based on the difference of the methods, this may need to be interpreted differently. The two commonly used types are the Prechtl $[9,10]$ and the Hadders-Algra methods [14]. The Hadders-Algra method classifies the GMs as normal (optimal or suboptimal) or abnormal (mildly or definitely) [14]. In the mildly abnormal category, the movements show a lack of variability and complexity, but the FMs are still present. We note that the "mildly abnormal" category in the Hadders-Algra method [14] would be considered normal with FMs present according to the Prechtl method $[9,10]$. In the definitely abnormal category, the movements again lack variability and complexity, but there are no FMs and there may be CS movements present. Thus, the abnormal category for the Hadders-Algra method is quite broad [14]. The definitely abnormal category is the only category with the absence of FMs.

This makes it challenging to compare it to the Prechtl method $[9,10]$ in terms of the GMs at 3 months as the Prechtl method $[9,10]$ is specific to determining if the FMs are present, absent, or abnormal.

\section{Context}

We considered the variability in the diagnosis of NE and so chose a baseline of at least a history and neurological examination for the diagnosis. We chose to look at all countries to be able to be as exhaustive as possible in our search.

Studies in the English language only were considered as there is no team member with adequate language skills to translate from any other language.

\section{Search strategy and databases searched}

A search of the literature covered the databases of MEDLINE, Embase, PsychINFO, Scopus, and CINAHL, to be inclusive of medicine, nursing, allied health professions, sociology, psychology, education, and social work. The General Movements Trust website was also searched [42].

The search strategy was phased, firstly created in Ovid MEDLINE using a combination of index terms and keywords around general movements, Prechtl, brain disease,
HIE, and perinatal asphyxia. An initial limited search of Ovid MEDLINE, Embase, and PsychINFO was undertaken to identify articles on the topic (see Additional file 3). There were no previous similar reviews identified. The text words contained in the titles and abstracts of relevant articles, and the index terms used to describe the articles from this limited search were then used to develop a more refined full search strategy in the second phase (Additional file 4).

This scoping review considered both experimental and quasi-experimental study designs including randomized controlled trials, non-randomized controlled trials, before and after studies, and interrupted time-series studies. Case reports, case series, case-control and crosssectional studies, and systematic reviews were considered. Studies published from at least 1970 were included. Prechtl first described GM in 1979, so we chose the date of 1970 to ensure all the related research would be captured $[9,15]$. The reference lists of articles were scanned, and experts in the infant developmental field were consulted to identify studies relevant to our topic.

\section{Study selection}

EndNote X9 (Clarivate Analytics, PA, USA) was used for citation collation. Duplicates were removed manually. Covidence (Covidence Systematic Review Software, Veritas Health Innovation, Melbourne, Australia) was used for screening by two independent reviewers (JS and ML). Disagreements were resolved through a third reviewer (RB). The results of the search were reported in a Preferred Reporting Items for Systematic Reviews and Metaanalyses extension for scoping reviews [43].

Ethical approval was not required as this was a scoping review and did not contain information directly identifying patients or content requiring patient consent. We conducted our bibliographic database searches between April 30, 2019, and March 30, 2020. The reference lists of all full-text relevant studies that were identified were hand-searched for additional relevant studies. Citations were identified and duplicates removed and screened by two independent reviewers (JS, ML). Relevant studies were identified for full-text review and searched for via Google Scholar, institutional journal access, e-Resources, and databases sites. Any disagreements that arose between the reviewers at each stage of the study selection process were resolved through discussion. A third reviewer $(\mathrm{RB})$ was the final arbitrator for any unresolved disagreements.

From the full texts, articles were selected for further review that met most of the inclusion and exclusion criteria. From these, articles were identified that fully met all the criteria. The results of the search were reported in a Preferred Reporting Items for Systematic Reviews and 
Meta-analyses (PRISMA-ScR) flow diagram [44]. Each article was independently reviewed and assessed by two of the authors (JS, ML). The data was extracted from articles using a data extraction tool developed by the reviewers. The format for the data extraction tool was modeled after being used by Kwong et al. in their 2018 review [16] (see Additional file 6). This decision was taken as that review aligned well with our study in describing the predictive ability of GM for later $\mathrm{CP}$, although not specifically to our population.

The distribution of the studies was determined by year of publication, as well as country of origin. These were important contextual factors as the older studies and the physical and human resources of each country may have posed limiting factors. A major consideration could have been the ability to obtain a representative portion of the population which affects the generalizability of the results. The study characteristics included information such as gender, GA, birth weight, and numbers of term and late-preterm neonates in the sample as well as the overall sample size, the number of neonates diagnosed with NE, and the number of cases of $\mathrm{CP}$.

\section{Results}

Following the searches, 903 citations were identified. The results of the search were reported here in a flow diagram (Fig. 1), adapted from the PRISMA-ScR [44] structure.

There were 25 studies $[13,45-68]$ that did not meet the full inclusion criteria but contained important information with regard to our topic. We placed these in an exclusion table (Additional file 4: Table 4 and 5). Of these 25 studies, 20 [13, 45-47, 49-52, 54, 55, 57-66] included late-preterm and term infants but did not delineate them as a specific group as it relates to their diagnosis of $\mathrm{NE}$ and their CP outcomes and when using GMA as a predictive tool. Additional file 4: Table 4 presents the summary of the characteristics of the excluded studies. There was a wide variety in their key characteristics. We summarized these characteristics here. These studies had a wide date range from 1997 to 2021 . They were mainly prospective studies [13, 45, 46, 48-50, 52-54, 57, 59, 60, 62-65] (16 of the 25), and the majority used clinical assessments only to identify infants at high risk [46, 49, 50, 52-54, 60, 61, 63-68] (14 of the 25). With regard to the GMA tools used, of the 25 studies, 23 used the Prechtl GMA and the other two used the Hadders-Algra method [48, 65]. In the study by Dekkers et al. [48], all the children with an abnormal score had either CP or another severe developmental delay.

For the age at which $\mathrm{CP}$ was diagnosed, 16 of the excluded studies used the same criteria as we did in this study, that is, CP diagnosis by at least $2-3$ years $[13,46$, $48,49,53,54,56-61,63,64,66]$. For the method of CP diagnosis, a variety of standardized assessments were used, with the most frequent being by Amiel-Tison and Grenier [69] (5 of the 25) [13, 47, 54, 60, 63] and Touwen Infant Neurological Examination (TINE) [70] (5 of the 25) $[46,47,49,54,64]$ with other assessments [71-81] detailed in Additional file 4: Table 4.

Eight studies either used non-standardized methods or did not clearly state their method $[45,50,51,56,57,59$, 62, 65].

Additional file 4: Table 5 presents the summary of the key findings of these excluded studies and reasons for their exclusion. These findings showed that in highrisk infants, including those with NE, GMA is a strong predictor of CP [45], especially when used in the fidgety period $[46,53,55,57,61,63,65,66]$. In 1997, Prechtl et al. [13] demonstrated that movement quality was important. Abnormal quality and absent fidgety movements, in a mixed group of preterm and term infants, predicted neurological abnormalities with a sensitivity of $96 \%$. The majority of these were diagnosed as CP. We see in our results that over time, this result has been repeatedly duplicated showing that CS [49] and absent fidgety [58] GM are highly predictive of CP. The trajectory of the GMA is more important as a predictor of CP [46].

The GMA is more sensitive than the traditional neurological examination [47, 49, 52], and the sensitivity increases with the combined use of other modalities such as electroencephalogram (EEG) [52], neuroimaging [62], HINE [27], and neuroimaging [58].

For these excluded studies, sensitivity values were as high as $100 \%[45,46,49,54,57]$ and specificity similar close to or at $100 \%[45,53,58,61,66]$. We contacted the authors, Solemani et al. [61] and Goyen et al. [53], of the studies closest to our inclusion criteria. Solemani et al. [61] delineated their populations by NE and by GA, but their outcome was reported as "neurodevelopmental outcomes" and not CP. They reported to us that they did not specifically report $\mathrm{CP}$ and so could not be included for us. Similarly, Goyen et al. [53] reported their outcomes not specifically divided for preterm versus term as their aim was to describe the NICU experience. We were unable to include this study in our final count. Both studies, however, reported on the high predictive validity of the Prechtl GMA at 3 months as it relates to neurodevelopmental outcomes at 2-3 years, and the Goyen et al. [53] study specifically for $\mathrm{CP}$ at that age. Nine of the excluded studies $[13,52,55,58-62,66]$ reported on positive predictive value (PPV) and negative predictive value (NPV) with some studies reporting PPV as high as $98 \%$ when used in combination with HINE and neuroimaging [58] or $75 \%$ with combined with EEG and ERP [66]. Negative predictive value was reported close to $98.31 \%$ [66] or at $100 \%$ [54]. Themes for the limitations identified by the 


\section{PRISMA 2009 Flow Diagram}

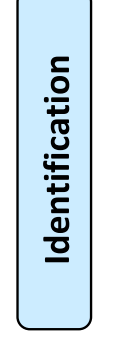

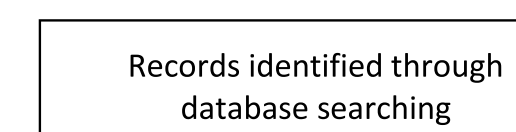
database searching

$$
n=873
$$

\section{Additional records identified} through other sources

$$
\mathrm{n}=30
$$
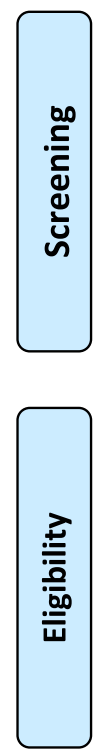

Full-text articles

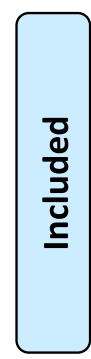
excluded, with reasons

$$
n=25
$$

8 Late-preterm outcomes not delineated

6 Late-preterm and term

diagnosis and outcome not delineated

$6 \mathrm{CP}$ diagnosis not delineated instead a combined outcome given

2 Term diagnosis not delineated

2 Protocol/Pilot study

1 Neonatal encephalopathy not well defined
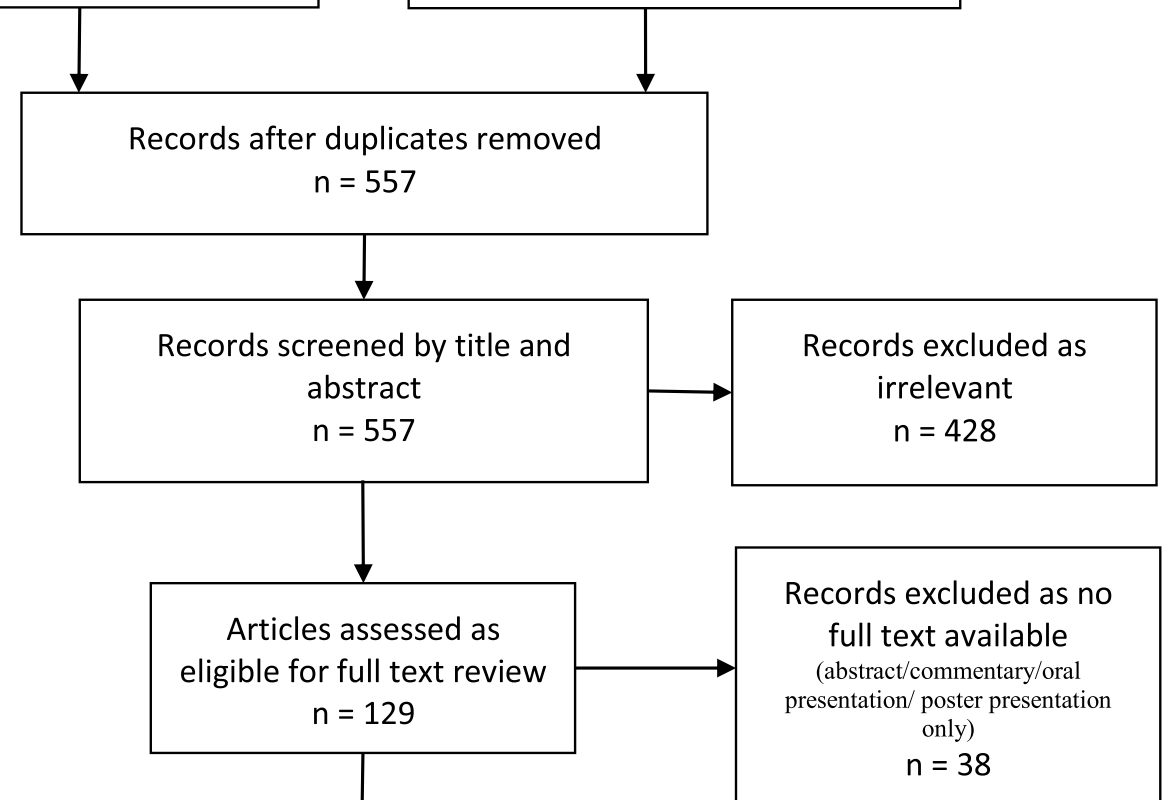
for eligibility

$$
\mathrm{n}=91
$$

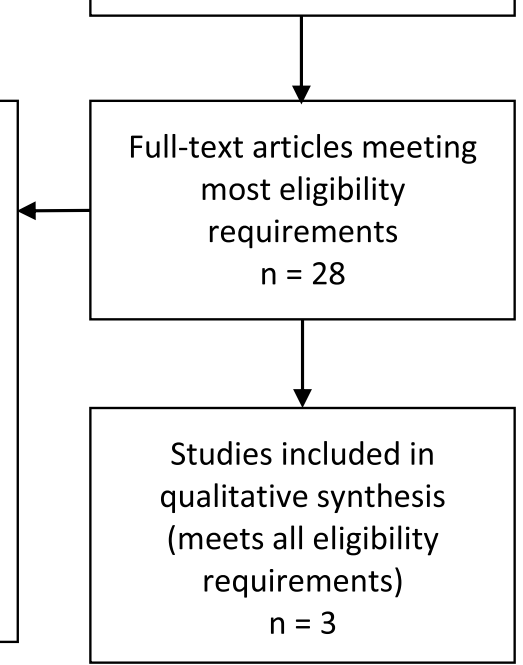

Records excluded as irrelevant $n=428$

Full-text articles excluded, with reasons

$$
n=63
$$

20 Wrong patient population

8 Wrong study design

7 No GM done

6 Wrong outcomes

6 Not in English

article

3 SR

3 Not a research article

2 Protocol article

1 Exclusive computer-based

assessment

1 Wrong indication

1 Wrong intervention
5 URL present but cannot access

Fig. 1 Flow chart for the selection of articles at different phases of the scoping review 
authors can be summarized as limited external validity due to small population size $[48-50,56,58,63,64,66]$ selection bias related to recruitment from high-risk populations $[13,51,58]$, and practice variation between sites $[45,54,57]$. The most common reasons for the exclusion of these studies were failure to delineate their participants for the diagnosis of NE, most quoting their participants as high-risk infants, or not delineating their GA into the groups relevant to our questions (late-preterm and term) [45, 49-54, 57, 58, 60, 62-66].

Only three articles, therefore, Ferrari et al. [82] Glass et al. [83], and Prechtl et al. [84], were identified as meeting the selection criteria and were included in the final review. The results of the search were reported here in a flow diagram (Fig. 1). The final studies included one prospective cohort study from the USA [83], and the other two were case series $[82,84]$ from Italy. The total number of participants was only 118 term neonates $(58,34$, and 26 participants); none included late-preterm neonates. Neonatal encephalopathy was reported as a single group by Glass et al. [83] and Ferrari et al [82] but divided into mild-moderate and severe by Prechtl et al. [84]. The high-risk groups in the cohort study [83] used a combination of clinical diagnosis, EEG, and MRI where possible to identify the NE population while for both case studies, NE was identified by history only. The GMA used by all three studies was Prechtl. Additional file 4: Table 6 presents the characteristics of these three studies. The prospective cohort study was published in 2021 [83] and reflected data collected within the previous 6 years, which would be reflective of the current management practices, especially as they did report on $68 \%$ of their population having received therapeutic hypothermia. Both of the case studies were published more than 5 years ago with data collected in excess of 15 years ago during which time the standard of care for NE is likely to have been different from the current practices. All three studies reported on sensitivity, specificity, PPV, and NPV.

A variety of standardized tools were used for $\mathrm{CP}$ diagnosis between the three studies. Additional file 4: Table 7 details the key findings and the outcomes evaluated, with the limitations identified by the authors. Glass et al. [83] reported on the absence of FM for the prediction of CP. Their findings were higher for the specificity 96-98\% than sensitivity $29-50 \%$ for any $\mathrm{CP}$ and for moderate to severe CP, respectively. Notably, their NPV was $90 \%$ for any $\mathrm{CP}$ and $98 \%$ for moderate to severe $\mathrm{CP}$, indicating that the presence of FM at 3 months is a strong indicator of an infant at low risk for a later diagnosis of $\mathrm{CP}$, especially in the moderate to severe category. Although not part of the specific aims of our study, in the study by Glass et al. [83], it was significant that when they combined the Prechtl GMA and MRI findings, the NPV increased to
$100 \%$. In the study by Ferrari et al. [82] they reported that the presence of any CS movements between term and 4 to 5 months post-term had a sensitivity of $100 \%$ and a specificity of $68.7 \%$, with a PPV $100 \%$ and a NPV of $78.3 \%$ for predicting CP. In the oldest study by Prechtl et al [84], the predictive ability in terms of the timing of the GMA was determined, that is, if done early, in the first 2 weeks of life versus late assessments between 15 and 22 weeks of life. Their findings were: sensitivity $100 \%$ and specificity $46.2 \%$, with PPV $65.0 \%$ and NPV $100 \%$ for the early assessments, compared to late assessments with $84.6 \%$ across the board for sensitivity, specificity, PPV, and NPV. Neither of the case studies included infants receiving therapeutic hypothermia for NE which was not yet the standard of care. Ferrari et al. [82] identified selection bias as a limitation, where mild HIE as a contributor to NE may have been underrepresented due to these infants not being referred for evaluation. Prechtl et al. [84] did not state their limitations.

\section{Risk of bias}

Even though this was a scoping review and did not require the critical appraisal of the three included articles, the critical appraisal tool for JBI $[85,86]$ helped to assess the quality of the articles and identify the differences and similarities between these two case studies. These main points are summarized here, and details are presented in Additional file 5: Table 8.

The quality of evidence derived from a review is largely dependent on the quality of the studies included.

This observational prospective cohort study by Glass et al. scored $100 \%$ in 10 of 11 questions [83]. This therefore assesses this study to be of high quality. The single question for which the study did not score $100.0 \%$ was that of the strategies used to address incomplete followup. Incomplete follow-up may result in selection bias. According to the JBI method, it is important that all the outcomes are assessed and participants with unequal follow-up periods must be taken into account in the analysis. For this study, patients with incomplete followup were not analyzed. If the analysis was not statistically feasible, this was not stated in the study.

Neither of the case studies scored $100 \%$ on all ten questions. The two case studies scored $100 \%$ for six of the ten questions on the checklist. These questions assess the two included case studies as being moderate-quality case series as there were limitations. They had good scores for using valid methods for the identification of the condition for all participants, having clear reporting of the demographics of the participants in the study, as well as, having clear clinical information of the participants. The outcomes of the cases were clearly reported for both studies. They also had clear reporting of the presenting 
site demographic information and used appropriate statistical analysis.

According to the JBI method, for the study participants, the authors should provide clear exclusion criteria. These inclusion and exclusion criteria should be specified with sufficient details and all the necessary information critical to the study. While Ferrari et al. [82] did fulfill these criteria, of note, Prechtl et al. [84] did not state their exclusion criteria, so this may limit the generalizability of the results. For good-quality case series, the study should clearly describe the method of measurement of the condition. This should be done in a standard (i.e., same way for all patients) and reliable (i.e., repeatable and reproducible results) way. The clinical condition for our study is NE. Both case studies listed a number of criteria for possible inclusion for NE but did not state the number or combination of these criteria required for the diagnosis and so scored $0.0 \%$ for this question. They did use a standard, albeit different, method for NE severity, with Ferrari et al. [82] used the Sarnat staging [7] while Prechtl et al. [84] used the Levene method [87]. With regard to the consecutive inclusion, studies that indicate a consecutive inclusion are more reliable than those that do not. Neither of our included studies stated clearly if they did consecutive inclusion of every neonate meeting the inclusion criteria, at their institutions, during the identified periods. Thus, they both scored $0.0 \%$ for this. Along a similar vein, the completeness of a case series contributes to its reliability. Studies that indicate a complete inclusion are more reliable than those that do not. Neither Ferrari et al. [82] nor Prechtl et al. [84] clearly stated that they included all the patients in their studies and scored $0.0 \%$ for this question.

The biases include selection, information, and sampling variation. Selection bias is typical of case series as it is a choice of a series of patients with a particular illness (NE), and a suspected linked outcome (CP) [88]. Selection bias limits the generalizability of the results. Information bias is less in retrospectively collected data as it is determined by what is already documented in the medical chart. These three studies were prospectively collected data making them susceptible to information bias. With regard to sampling variation, the precise determination of the rate of a disease, other than by chance, requires a large sample size. All of the included studies can be described as employing small sample sizes, and Glass et al. [83] had the highest number of participants at 58, while Ferrari et al. [82] had 34 cases and Prechtl et al. [84] had 26 cases with a follow-up period of over 3 to 4 years. Sample size may have been limited by the collection method as no study stated if they were inclusive of every neonate meeting the inclusion criteria, at their institutions, during the identified periods.

\section{Discussion}

The scoping review methodology provided valuable insight into the current limited state of knowledge on the use of the GMA in the term neonate diagnosed with NE to predict CP by the age of 2-3 years. In fact, in the late preterm infant with NE, there was no evidence that met our inclusion criteria. Furthermore, the current evidence for our population was until recently, with the study by Glass et al. [83] derived from two case studies which do not constitute a level of evidence on which we can base definite recommendations. Our scoping review results highlight the need for more specific, higher-quality research in this area.

From the review, we were able to glean some important insights into the use of the GMA in our population. In the study by Glass et al. [83], infants with absent FMs at 3 months should be monitored closely as they are at high risk for a diagnosis of moderate to severe CP. The high NPV indicate that if FMs are present at the 3-month assessment of the GMA, the outcome is unlikely to be moderate to severe $\mathrm{CP}$. In addition, a normal neonatal MRI in combination with a normal GMA at 3 months in a term high-risk infant is likely associated with a low risk for moderate to severe $\mathrm{CP}$. This is reassuring advice that can be given to parents. In both case studies, the presence of the CS movement pattern of the GMA does correlate with the prediction of $\mathrm{CP}$ by 2 years of age. Prechtl et al.[84] noted that NE has an effect on spontaneous movements in term neonates, be it transient or persistent. Early assessments may be unable to differentiate between abnormal spontaneous movements that may be transient from those that will persist and eventually be associated with CP. Early assessments do not give as good predictive values as later assessments; therefore, the trajectory of the GMA may be a more significant indicator of outcomes than a solitary assessment [46, 84].

\section{Limitations of the included studies}

Our findings support the role of the GMA as a good tool for the prediction of $\mathrm{CP}$ for those infants born at term with NE. There are however limitations to consider including the following: two of the three publications were case series, the variability in the NE definitions, the date of the publications identified and neither study contained neonates treated with the now standard of care, therapeutic hypothermia. Another important limitation is the low number of studies meeting our inclusion criteria.

Firstly, internal validity is likely to be low, as occurs especially in case series, since there are no comparator groups exposed to a similar array of variables. External validity would similarly be limited. Since this scoping 
review only represents level IV evidence [89], it reveals the need for future research in this area since it suggests that neonates with NE, at least those at term, may benefit from follow-up assessments with the GMA to help earlier identification of CP.

Secondly, in terms of the definition of NE, for the study by Ferrari et al. [82], there were some differences in the way NE was defined. In general, the standard accepted criteria that define NE were used, but they stated that study participants had different combinations of the NE criteria. Evidence shows that the etiology $[8,16]$ of encephalopathy as well as its severity may influence the outcomes [8]. Although the severity of NE (that is, mild, moderate, or severe reflected by the Sarnat stage [7]) was assessed in the study, no further differentiation of severity as it related to the predictive ability for $\mathrm{CP}$ was done. This may have been due to the small sample size of the study $(n=34)$ and the inevitable decreased power that would have resulted from subdivisions. Prechtl et al. [84] also subdivided the NE diagnosis into mild to moderate $(n=13)$ and severe $(n=13)$ NE. Similar to Ferrari et al. [82], the outcomes were not reported according to these NE subdivisions.

Thirdly, in terms of the timing of the publications, both of the case studies were more than 5 years old. Management has changed over time, and updated data in this evolving area would be beneficial. It is however interesting that the older study by Prechtl et al. [84] in 1993 supports the same later findings of the Ferrari et al. [82] study in 2011 with respect to the predictive ability of the GMA. This lends support to the reliable role of the GMA in the identification of those children born at term with $\mathrm{NE}$ who are at risk for $\mathrm{CP}$.

Fourthly, neither of the case studies seemed to have been done in neonates treated with therapeutic hypothermia, which is the current standard of care $[3,4]$. We are therefore uncertain if therapeutic hypothermia changes the quality of the GM, and if it does change, how long might this persist. Information like this is important to inform the timing of the early GMA post-therapeutic hypothermia intervention. Similar consideration had to be done for identifying the optimal window for cranial MRI in neonates treated with therapeutic hypothermia [90]. This lends credence to the gap in research in this area of NE and its association with CP.

Finally, low sample sizes in all the studies limit the power of the studies.

\section{Strengths and limitations of this review}

The strength of our review primarily lies in the scoping review methodology that we chose. This method was advantageous as it facilitated an exhaustive search of the literature to define the current extent of knowledge and so allowed the research gaps to be identified.

We appreciated that there were several limitations. Firstly, we excluded studies that were not in English. This may be significant as there were only three studies identified in the review that met the established inclusion criteria. Therefore, with this limited number, any additional studies may have impacted our results. Secondly, the ages of the studies are of concern. Only one study was published recently in 2021 [83] with participants recruited between the years 2015 to 2017 and included patients that received therapeutic hypothermia. The study that was published in 2011 by Ferrari et al. [82], recruited participants between 2003 and 2006. The cohort of the Prechtl study was recruited between 1985 and 1989. Medical management has evolved since these two case studies to include strategies such as therapeutic hypothermia [3, 4]. The impact of this on our results is unknown. Lastly, we recognize that using a cutoff of CP diagnosis by $2-3$ years of age constitutes a limitation for a number of reasons. Mild motor impairments may resolve with early intervention and not be eventually classified as a $\mathrm{CP}$ diagnosis. On the other hand, even milder forms of CP may not be identified early by $2-3$ years of age and become apparent when more higher function motor tasks are required at older ages.

\section{Suggestions for further research}

This review elucidates multiple potential areas for research.

\section{Quantitative research}

Meta-analysis - a meta-analysis of the data was not possible due to the minimal number of studies available. As more data becomes available, more accurate suggestions, for the use of the GMA in our population, can be made.

Prospective study-to provide higher quality evidence, research would preferably be a multi-center prospective cohort study, with matched low-risk controls. Standardization to the Prechtl GMA and the same assessment for $\mathrm{CP}$ at the same postnatal age would also add to the quality of the study. A study looking at the use of the GMA and its detailed qualitative analysis, the Motor Optimality Score (MOS), in our population of term and late-preterm infants diagnosed with NE is needed. Medical management has changed since the era of the cohorts in two of the three studies included in this review. More studies with the link of the GMA with respect to NE severity, etiology, and contemporary management would be of benefit. Alkan et al. 2021 [91] published a case-control study looking retrospectively at the motor repertoire in term infants with HIE at 3-5 months post-term using the MOS. They found that the total MOS scores were lower 
than that of their neurotypical counterparts. They also found that the higher the severity of HIE, the lower the MOS score. This study provides up-to-date further support for the need for a large prospective cohort study in this area.

Predictive ability of GMA in CP severity in our population-determination of the predictive ability of the GMA, including the MOS, for the degree of functionality in $\mathrm{CP}$, such as that determined by the Gross Motor Function Classification System (GMFCS) [74] scoring system would be beneficial for implementation of early intervention strategies.

Optimal timing of an early assessment-since the trajectory of the GM may provide significant clues to neurodevelopmental outcomes more research is needed in this area. Transient effects on the GM may result from medical management such as therapeutic hypothermia and medications including sedatives and anticonvulsants. The optimal timing of the first assessment in this population requires further elucidation.

\section{Qualitative research}

Parental counseling and anticipatory interventionparental perspectives on the use of the GMA as a predictor of neurodevelopmental outcomes in our population would be desirable for future research. This would influence counseling by the medical teams with regard to reassurance or the need for early intervention and the extent of neurodevelopmental follow-up.

\section{Conclusion}

In term infants with encephalopathy, the predictive ability of the GMA is not as reliable when performed early versus later (at 15-22 weeks of age). The finding of normal GMs at 3 months of age is reassuring that a highrisk neonate is unlikely to develop moderate to severe CP. The finding of CS GM is a predictor for the diagnosis of $\mathrm{CP}$ by 2 years of age in the term population with NE.

Additionally, there are no existing studies specific to the application of the GMA in late-preterm infants with NE.

The deficit of high-quality research limits the applicability, so the GMA should not be used in isolation when assessing these populations. The evidence for this is limited by few studies and a lack of high-quality research. The evidence is lacking for the utilization of the GMA in these populations treated with therapeutic hypothermia. Furthermore, of possibly greater potential applicability is the inclusion of the MOS as a predictor of CP $[92,93]$ in term and late preterm infants with NE when treated with therapeutic hypothermia.

\section{Abbreviations}

BW: Birth weight; Cl: Confidence interval; CP: Cerebral palsy; CS: Cramped synchronized; EEG: Electroencephalogram; ELBW: Extremely low birth weight; ERP: Event-related potential; FM: Fidgety movements; GA: Gestational age; GM: General movements; GMA: General Movements Assessment; GMFCS: Gross Motor Function Classification System; HIE: Hypoxic-ischemic encephalopathy; HINE: Hammersmith Infant Neurological Examination; JBI: Joanna Briggs Institute; LR: Likelihood ratio; LBW: Low birth weight; MOS: Motor Optimality Score; Movement-ABC: Movement Assessment Battery for Children; MRI: Magnetic resonance imaging; NAPI: Neurobehavioral Assessment of the Preterm Infant; NBW: Normal birth weight; NE: Neonatal encephalopathy; NPV: Negative predictive value; n.s.: Not stated; PCC: Participant, concept, context; PMA: Postmenstrual age; PR: Poor repertoire; PPV: Positive predictive value; PRISMA-SCR: Preferred Reporting Items for Systematic Reviews and Meta-analyses extension for scoping review; TIMP: Test of Infant Motor Performance; TINE: Touwen Infant Neurological Examination; SD: Standard deviation; US: Ultrasound; VLBW: Very low birth weight.

\section{Supplementary Information}

The online version contains supplementary material available at https://doi. org/10.1186/s13643-021-01765-8.

Additional file 1. Protocol for GMA and NE in CPR2.

Additional file 2. Proposal.

Additional file 3. Search strategies.

Additional file 4. Tables for excluded and included studies.

Additional file 5. Critical appraisal.

Additional file 6. Tables for data extraction.

\section{Acknowledgements}

This review contributed to a Master in Healthcare Quality degree for JS. The authors would also like to acknowledge the librarians that assisted with this research project, namely from the Sunnybrook R. lan MacDonald Library, Henry Lam, and Reena Besa, as well as the librarians Paola Durando and Sandra McKeown of the Bracken Health Sciences Library, Queen's University.

\section{Authors' contributions}

Judy Seesahai: Principal Author (PA); substantial contributions to the research design; acquisition, analysis, and interpretation of the data; and drafting of the paper. Maureen Luther: contribution to the acquisition, analysis, and interpretation of the data and involved in the revisions to the paper. Paige Terrien Church: substantial contributions to the research design, analysis and interpretation of the data, and drafting of the paper. Patricia Maddalena: contribution to the acquisition, analysis, and interpretation of the data and involved in the revisions to the paper. Thomas Rotter: substantial contributions to the research design and review of the paper. Elizabeth Asztalos: substantial contributions to the research design; acquisition, analysis, and interpretation of the data; and drafting of the paper. Rudaina Banihani: Principal Investigator (PI) and corresponding author; substantial contributions to the research design; acquisition, analysis, and interpretation of the data; and drafting of the paper. The authors read and approved the final manuscript.

\section{Funding}

There is no funding required for this review.

\section{Availability of data and materials}

Data sharing is not applicable to this article as no datasets were generated or analyzed during the current study. Materials during the current study are available from the corresponding author on reasonable request. 


\section{Declarations}

\section{Ethics approval and consent to participate}

Ethical approval was not required as this is a scoping review of the literature and did not contain information directly identifying patients or content requiring patient consent.

\section{Consent for publication}

Not applicable.

\section{Competing interests}

The authors declare that they have no competing interests.

\section{Author details}

${ }^{1}$ Sunnybrook Health Sciences Centre, University of Toronto, Toronto, ON, Canada. ${ }^{2}$ Department of Pediatrics, Division of Neonatal-Perinatal Medicine, University of Toronto, Toronto, Canada. ${ }^{3}$ Queen's University, Kingston, ON, Canada. ${ }^{4}$ Newborn \& Developmental Paediatrics, Sunnybrook Health Science Centre, 2075, Bayview Ave., Toronto, ON M4N 3M5, Canada.

Received: 14 August 2020 Accepted: 20 July 2021

Published online: 12 August 2021

\section{References}

1. Rosenbaum P, Paneth N, Leviton A, Goldstein M, Bax M, Damiano D, et al. A report: the definition and classification of cerebral palsy April 2006. Dev Med Child Neurol Suppl. 2007;109(suppl 109):8-14.

2. American College of Obstetricians and Gynecologists. Neonatal Encephalopathy and Neurologic Outcome, Report of the American College of Obstetricians and Gynecologists'Task Force on Neonatal Encephalopathy. Obstet Gynecol. 2014;123(4):896-901.

3. Glass HC. Hypoxic-ischemic encephalopathy and other neonatal encephalopathies. Continuum Lifelong Learn Neurol. 2018;24(1):57-71.

4. Co F. Newborn Hypothermia and neonatal encephalopathy. Pediatrics. 2014;133(6):1146-50

5. Tagin MA, Woolcott CG, Vincer MJ, Whyte RK, Stinson DA. Hypothermia for neonatal hypoxic ischemic encephalopathy: an updated systematic review and meta-analysis. Arch Pediatr Adolesc Med. 2012;166(6):558-66.

6. Staub K, Baardsnes J, Hébert N, Hébert M, Newell S, Pearce R. Our child is not just a gestational age. A first-hand account of what parents want and need to know before premature birth. Acta Paediatr. 2014;103(10):1035-8.

7. Sarnat HB, Sarnat MS. Neonatal encephalopathy following fetal distress. A clinical and electroencephalographic study. Arch Neurol. 1976;33(10):696-705.

8. Miller SP, Latal B, Clark H, Barnwell A, Glidden D, Barkovich AJ, et al. Clinical signs predict 30-month neurodevelopmental outcome after neonatal encephalopathy. Am J Obstet Gynecol. 2004;190(1):93-9.

9. Prechtl HF, Fargel JW, Weinmann HM, Bakker HH. Postures, motility and respiration of low-risk pre-term infants. Dev Med Child Neurol. 1979;21(1):3-27.

10. De Vries JI, Visser GH, Prechtl HF. The emergence of fetal behaviour I Qualitative aspects. Early Hum Dev. 1982;7(4):301-22.

11. Ferrari F, Einspieler $C$, Prechtl H, Bos A, Cioni G. Prechtl's method on the qualitative assessment of general movements in preterm, term and young infants: Mac Keith Press; 2004.

12. Bosanquet M, Copeland L, Ware R, Boyd R. A systematic review of tests to predict cerebral palsy in young children. Dev Med Child Neurol. 2013;55(5):418-26.

13. Prechtl HF, Einspieler C, Cioni G, Bos AF, Ferrari F, Sontheimer D. An early marker for neurological deficits after perinatal brain lesions. Lancet. 1997;349(9062):1361-3.

14. Hadders-Algra M. General movements: a window for early identification of children at high risk for developmental disorders. J Pediatr. 2004;145(2 Suppl):S12-8

15. Novak I, Morgan C, Adde L, Blackman J, Boyd RN, Brunstrom-Hernandez $J$, et al. Early, Accurate diagnosis and early intervention in cerebral palsy: advances in diagnosis and treatment. JAMA, Pediatr. 2017;171(9):897-907.
16. Kwong AKL, Fitzgerald TL, Doyle LW, Cheong JLY, Spittle AJ. Predictive validity of spontaneous early infant movement for later cerebral palsy: a systematic review. Dev Med Child Neurol. 2018;22:22.

17. Noble Y, Boyd R. Neonatal assessments for the preterm infant up to 4 months corrected age: a systematic review. Dev Med Child Neurol. 2012;54(2):129-39.

18. Zuk L. Fetal and infant spontaneous general movements as predictors of developmental disabilities. Dev Disabil Res Rev. 2011;17(2):93-101.

19. Darsaklis V, Snider LM, Majnemer A, Mazer B. Predictive validity of Precht's method on the qualitative assessment of general movements: a systematic review of the evidence. Dev Med Child Neurol. 2011;53(10):896-906.

20. Burger M, Louw QA. The predictive validity of general movements-a systematic review. Europ J Paediatr Neurol. 2009;13(5):408-20.

21. Spittle AJ, Doyle LW, Boyd RN. A systematic review of the clinimetric properties of neuromotor assessments for preterm infants during the first year of life. Dev Med Child Neurol. 2008;50(4):254-66.

22. Hadders-Algra M. Evaluation of motor function in young infants by means of the assessment of general movements: a review. Pediatr Phys Ther. 2001;13(1):27-36.

23. Santos RS, Araújo AP, Porto MAS. Early diagnosis of abnormal development of preterm newborns: assessment instruments. J Pediatr (Rio J). 2008;84(4):289-99.

24. Valencia A. Discriminative and predictive validity of the general movements assessment: a systematic review. Prospero Int Prospective Regist of Syst Rev. 201814

25. Kwong A, Fitzgerald T, Spittle A, Cheong J, Doyle L, Einspieler C. A systematic review of the predictive validity of observational early infant motor assessments for subsequent cerebral palsy. Prospero Int Prospective Regist Syst Rev. 2016.

26. Seesahai J, Luther M, Rhoden CC, Church PT, Asztalos E, Banihani R. The general movements assessment in term and late-preterm infants diagnosed with neonatal encephalopathy, as a predictive tool of cerebral palsy by 2 years of age: a scoping review protocol. Syst Rev. 2020;9(1):154

27. Romeo DM, Ricci D, Brogna C, Mercuri E. Use of the Hammersmith Infant Neurological Examination in infants with cerebral palsy: a critical review of the literature. Dev Med Child Neurol. 2016;58(3):240-5.

28. Harris SR. Early neuromotor predictors of cerebral palsy in low-birthweight infants. Dev Med Child Neurol. 1987;29(4):508-19.

29. Piper C. System for the unified management, assessment, and review of information (SUMARI). J Med Libr Assoc. 2019;107(4):634.

30. Burnsed J, Zanelli SA. Neonatal therapeutic hypothermia outside of standard guidelines: a survey of US neonatologists. Acta Paediatr. 2017;106(11):1772-9.

31. Group ACPR, Badawi N, Balde I, Goldsmith S, Karlsson P, Mclntyre S, et al. Australia and the Australian Cerebral Palsy Register for the birth cohort 1993 to 2006. Dev Med Child Neurol. 2016;58:3-4.

32. Australia Cerebral Palsy Register. Australian cerebral palsy register report 2018. Canberra: the Australian Cerebral Palsy Register Group. 2018.

33. Granild-Jensen JB, Rackauskaite G, Flachs EM, Uldall P. Predictors for early diagnosis of cerebral palsy from national registry data. Dev Med Child Neurol. 2015;57(10):931-5.

34. Lindström K, Bremberg S. The contribution of developmental surveillance to early detection of cerebral palsy. Acta Paediatr. 1997;86(7):736-9.

35. Burns Y, O'Callaghan M, Tudehope D. Early identification of cerebral palsy in high risk infants. J Paediatr Child Health. 1989;25(4):215-9.

36. LockTM, Shapiro BK, Ross A, Capute AJ. Age of presentation in developmental disability. J Dev Behav Pediatr. 1986;7(6):340-5.

37. Banihani R, Church PT, Luther M, Maddalena P, Asztalos E. Neonatal encephalopathy. Follow-Up for NICU Graduates. Cham: Springer; 2018. p. $155-78$.

38. Kyriakopoulos P, Oskoui M, Dagenais L, Shevell MI. Term neonatal encephalopathy antecedent cerebral palsy: a retrospective populationbased study. Europ J Paediatr Neurol. 2013;17(3):269-73.

39. Rosenbloom L. Dyskinetic cerebral palsy and birth asphyxia. Dev Med Child Neurol. 1994;36(4):285-9.

40. Stanley FJ, Blair E, Hockey A, Petterson B, Watson L. Spastic quadriplegia in Western Australia: a genetic epidemiological study. I: Case population and perinatal risk factors. Dev Med Child Neurol. 1993;35(3):191-201.

41. Trevethan R. Sensitivity, specificity, and predictive values: foundations, pliabilities, and pitfalls in research and practice. Front Public Health. 2017:5:307. 
42. Einspieler C. General movements trust. The Prechtl General Movements Assessment [Internet]. Austria. 2009.

43. Tricco AC, Lillie E, Zarin W, O'Brien KK, Colquhoun H, Levac D, Moher D, Peters MD, Horsley T, Weeks L, Hempel S. PRISMA extension for scoping reviews (PRISMA-ScR): checklist and explanation. Annals Intern Med. 2018;169(7):467-73.

44. Moher D, Liberati A, Tetzlaff J, Altman DG. Preferred Reporting Items for Systematic Reviews and Meta-Analyses: the PRISMA statement. Int I Surg. 2010;8(5):336-41.

45. Adde L, Rygg M, Lossius K, Oberg GK, Stoen R. General movement assessment: predicting cerebral palsy in clinical practise. Early Hum Dev. 2007;83(1):13-8.

46. Brogna C, Romeo DM, Cervesi C, Scrofani L, Romeo MG, Mercuri E, et al. Prognostic value of the qualitative assessments of general movements in late-preterm infants. Early Hum Dev. 2013;89(12):1063-6.

47. Cioni G, Prechtl HF, Ferrari F, Paolicelli PB, Einspieler C, Roversi MF. Which better predicts later outcome in fullterm infants: quality of general movements or neurological examination? Early Hum Dev. 1997;50(1):71-85

48. Dekkers L, Janssen A, Steiner K, Maas-van Schaijk N, Akkermans R, de Swart $B$, et al. Individual longitudinal neurodevelopmental trajectories of children treated with hypothermia for perinatal asphyxia from 3 months to 5 years of age. Res Dev Disabil. 2020;102:103659.

49. Dimitrijevic L, Bjelakovic B, Colovic H, Mikov A, Zivkovic V, Kocic M, et al. Assessment of general movements and heart rate variability in prediction of neurodevelopmental outcome in preterm infants. Early Hum Dev. 2016;99:7-12

50. Einspieler C, Yang H, Bartl-Pokorny KD, Chi X, Zang FF, Marschik PB, et al. Are sporadic fidgety movements as clinically relevant as is their absence? Early Hum Dev. 2015;91(4):247-52.

51. Einspieler C, Bos AF, Krieber-Tomantschger M, Alvarado E, Barbosa VM Bertoncelli $\mathrm{N}$, et al. Cerebral palsy: early markers of clinical phenotype and functional outcome. J Clin Med. 2019:8(10):1616.

52. Feng J, Ruan Y, Cao Q, Chen Y, Liang X. General movements and electroencephalogram as a predictive tool of high risk neonatal neurodevelopmental outcome. 2017.

53. Goyen TA, Morgan C, Crowle C, Hardman C, Day R, Novak I, et al. Sensitivity and specificity of general movements assessment for detecting cerebral palsy in an Australian context: 2-year outcomes. J Paediatr Child Health. 2020;56(9):1414-8.

54. Guzzetta A, Belmonti V, Battini R, Boldrini A, Paolicelli PB, Cioni G. Does the assessment of general movements without video observation reliably predict neurological outcome? Europ J Paediatr Neurol. 2007;11(6):362-7.

55. Hadders-Algra M, Van den Nieuwendijk AWK, Maitijn A, van Eykern LA. Assessment of general movements: towards a better understanding of a sensitive method to evaluate brain function in young infants. Dev Med Child Neurol. 1997;39(2):88-98.

56. King AR, Machipisa C, Finlayson F, Fahey MC, Novak I, Malhotra A. Early detection of cerebral palsy in high-risk infants: translation of evidence into practice in an Australian hospital. J Paediatr Child Health. 2021:57(2):246-50

57. Morgan C, Crowle C, Goyen TA, Hardman C, Jackman M, Novak I, et al. Sensitivity and specificity of General Movements Assessment for diagnostic accuracy of detecting cerebral palsy early in an Australian context. J Paediatr Child Health. 2016:52(1):54-9.

58. Morgan C, Romeo DM, Chorna O, Novak I, Galea C, Del Secco S, et al. The pooled diagnostic accuracy of neuroimaging, general movements, and neurological examination for diagnosing cerebral palsy early in high-risk infants: a case control study. J Clin Med. 2019;8(11):1879.

59. Oberg GK, Jacobsen BK, Jorgensen L. Predictive value of General Movement Assessment for cerebral palsy in routine clinical practice. Phys Ther. 2015;95(11):1489-95.

60. Seme-Ciglenecki P. Predictive value of assessment of general movements for neurological development of high-risk preterm infants: comparative study. Croat Med J. 2003;44(6):721-7.

61. Soleimani F, Badv RS, Momayezi A, Biglarian A, Marzban A. General movements as a predictive tool of the neurological outcome in term born infants with hypoxic ischemic encephalopathy. Early Hum Dev. 2015;91(8):479-82.

62. Stoen R, Boswell $L$, de Regnier RA, Fjortoft T, Gaebler-Spira D, Ihlen E, et al. The predictive accuracy of the general movement assessment for cerebral palsy: a prospective, observational study of high-risk infants in a clinical follow-up setting. J Clin Med. 2019;8(11):1790.

63. Sustersic B, Paro-Panjan D. Assessment of general movements in relation to neurologic signs at age two years. Pediatr Neurol. 2008;39(2):108-12.

64. van lersel PA, Bakker SC, Jonker AJ, Hadders-Algra M. Does perinatal asphyxia contribute to neurological dysfunction in preterm infants? Early Hum Dev. 2010;86(7):457-61.

65. Yang H, Einspieler C, Shi W, Marschik PB, Wang Y, Cao Y, et al. Cerebral palsy in children: movements and postures during early infancy, dependent on preterm vs. full term birth. Early Hum Dev. 2012;88(10):837-43.

66. Zhang Q, Hu Y, Dong X, Tu W. Predictive value of electroencephalogram, event-related potential, and general movements quality assessment in neurodevelopmental outcome of high-risk infants. Appl Neuropsychol. Child. 2021:1-6.

67. Pouppirt NR, Martin V, Pagnotto-Hammitt L, Spittle AJ, Flibotte J, DeMauro SB. The General Movements Assessment in neonates with hypoxic ischemic encephalopathy. J Child Neurol. 2021:0883073820981515.

68. Toldo M, Varishthananda S, Einspieler C, Tripathi N, Singh A, Verma SK, et al. Protocol: Enhancing early detection of neurological and developmental disorders and provision of intervention in low-resource settings in Uttar Pradesh, India: study protocol of the GANESH programme. BMJ Open. 2020;10(11).

69. Chaudhari S, Deo B. Neurodevelopmental assessment in the first year with emphasis on evolution of tone. Indian Pediatr. 2006:43(6):527.

70. Touwen B. Neurological development in infancy. London: SIMP and Heinemann Medical. Philadelphia: Lippincott; 1976.

71. Bayley N. Bayley scales of infant development: Harcourt Brace. 1993.

72. Griffiths R. The abilities of babies: a study in mental measurement. 1954.

73. Johnson A. Prevalence and characteristics of children with cerebral palsy in Europe. Dev Med Child Neurol. 2002:44(9):633-40.

74. Palisano R, Rosenbaum P, Walter S, Russell D, Wood E, Galuppi B. Development and reliability of a system to classify gross motor function in children with cerebral palsy. Dev Med Child Neurol. 1997;39(4):214-23.

75. Gesell Institute of human development. Gesell Developmental Schedule. 1979.

76. Hempel MS. The neurological examination technique for toddler-age: thesis. University of Groningen; 1993.

77. Dubowitz LM, DubowitzV, Mercuri E. The neurological assessment of the preterm and full-term newborn infant: Cambridge University Press; 1999.

78. Illingworth RS. The development of the infant and young child: normal and abnormal . 9th ed. --. Edinburgh; Churchill Livingstone; 1987.

79. Brown T, Lalor A. The Movement Assessment Battery for Children-second edition (MABC-2): a review and critique. Phys Occup Ther Pediatr. 2009;29(1):86-103.

80. Johnson S, Moore T, Marlow N. Using the Bayley-III to assess neurodevelopmental delay: which cut-off should be used? Pediatr Res. 2014;75(5):670-4.

81. Sung IY, Kang W. Infant Neurological International Battery (INFANIB) as a predictor of neuromotor outcome in risk infants. J Korean Acad Rehabil Med. 1997:21(2):406-13.

82. Ferrari F, Todeschini A, Guidotti I, Martinez-Biarge M, Roversi MF, Berardi A, et al. General movements in full-term infants with perinatal asphyxia are related to Basal Ganglia and thalamic lesions. J Pediatr. 2011;158(6):904-11.

83. Glass HC, Li Y, Gardner M, Barkovich AJ, Novak I, McCulloch CE, et al. Early identification of cerebral palsy using neonatal MRI and General Movements Assessment in a cohort of high-risk term neonates. Pediatr Neurol. 2021;118:20-5

84. Prechtl HF, Ferrari F, Cioni G. Predictive value of general movements in asphyxiated fullterm infants. Early Hum Dev. 1993;35(2):91-120.

85. Moola S, Munn Z, Tufanaru C, Aromataris E, Sears K, Sfetcu R, et al. Chapter 7: Systematic reviews of etiology and risk. Joanna Briggs Institute Reviewer's Manual The Joanna Briggs Institute. 2017;5.

86. Moola S MZ, Tufanaru C, Aromataris E, Sears K, Sfetcu R, Currie M, Lisy K, Qureshi R, Mattis P, Mu P. Chapter 7: Systematic reviews of etiology and risk. In: Aromataris E, Munn Z (Editors). JBI Manual for Evidence Synthesis. JBI. 2020

87. Levene ML, Kornberg J, Williams T. The incidence and severity of post-asphyxial encephalopathy in full-term infants. Early Hum Dev. 1985:11(1):21-6. 
88. El-Gilany A. What is case series. Asp Biomed Clin Case Rep. 2018;1(01):10-5.

89. The centre for evidence-based medicine. Oxford centre for evidencebased medicine - levels of evidence. 2009.

90. Bednarek N, Mathur A, Inder T, Wilkinson J, Neil J, Shimony J. Impact of therapeutic hypothermia on MRI diffusion changes in neonatal encephalopathy. Neurology. 2012;78(18):1420-7.

91. Alkan H, Kahraman A, Mutlu A. Early spontaneous movements of infants with hypoxic-ischemic encephalopathy. Pediatr. 2021;33(1):18-22.

92. Bruggink JL, Cioni G, Einspieler C, Maathuis CG, Pascale R, Bos AF. Early motor repertoire is related to level of self-mobility in children with cerebral palsy at school age. Dev Med Child Neurol. 2009;51(11):878-85.
93. Einspieler C, Marschik PB, Bos AF, Ferrari F, Cioni G, Prechtl HFR. Early markers for cerebral palsy: insights from the assessment of general movements. Future Neurol. 2012;7(6):709-17.

\section{Publisher's Note}

Springer Nature remains neutral with regard to jurisdictional claims in published maps and institutional affiliations.
Ready to submit your research? Choose BMC and benefit from:

- fast, convenient online submission

- thorough peer review by experienced researchers in your field

- rapid publication on acceptance

- support for research data, including large and complex data types

- gold Open Access which fosters wider collaboration and increased citations

- maximum visibility for your research: over $100 \mathrm{M}$ website views per year

At BMC, research is always in progress.

Learn more biomedcentral.com/submissions 